\title{
Ha ancora senso fare oggi EDD
}

\author{
Rosario Maiorca
}

Divisione e Cattedra di Nefrologia, Spedali Civili e Università, Brescia

$\mathbf{M}$ sia consentito di esprimere il mio parere in modo informale.

Per me ha ancora senso fare oggi EDD. Negli anni scorsi, nel mio reparto, siamo arrivati ad avere più di 50 pazienti in EDD, e i risultati sono sempre stati altamente soddisfacenti. Ovviamente non penso che la EDD abbia virtù miracolose, e i risultati buoni sono fondati sulla selezione del paziente, sulla sua motivazione, sulla presenza di un partner, in genere, motivato, e sul fatto che prendere coscienza della propria malattia e della sua cura aiuta a curarsi meglio.

Dopo l'arrivo della dialisi peritoneale (CAPD), anche da noi, come in tutto il mondo, vi fu un calo della EDD, proporzionale al crescere della CAPD. Tuttavia, anche dopo l'introduzione della CAPD, un piccolo gruppo di pazienti iniziò la EDD e ancora oggi ne mettiamo 1-2 all'anno. Perché? Perché sono convinto che, una volta fatta scegliere al paziente la metodica dialitica da lui preferita, in base a scelta razionale e non emotiva, se ve ne sono le condizioni si dovrebbe sempre proporre, a quelli che preferiscono l'emodialisi, la EDD. Ciò è tanto più giusto adesso che abbiamo, almeno noi a Bre- scia, grosse difficoltà a reperire nuovi posti per emodialisi.

Siamo in attesa, come tutti sanno, della mitica emodialisi domiciliare giornaliera semplificata nelle attrezzature e nella tecnica. Anche oggi, quindi, si può intravedere la possibilità di un prossimo rilancio della EDD.

Rimango dunque molto favorevole alla EDD, come alle altre tecniche domiciliari. La mia idea è sempre stata che l'ideale sarebbe curare tutti i pazienti al loro domicilio, con la tecnica a loro preferita, una volta fatte salve le poche, strette indicazioni mediche per una o l'altra, di esse. L'altro mio convincimento è che tutte le metodiche vanno viste come trattamenti che si integrano, per cui si può benissimo cominciare con una e passare all'altra, quando necessario. In questa visione il primo trattamento migliore è quello che piace di più al paziente, possibilmente a basso costo, quindi possibilmente fatto a domicilio. I risultati vanno poi valutati non tanto sulla singola metodica ma su quelli generali del Centro, tanto in termini di mortalità che di morbidità.

maiorca@master.cci.unibs.it 